\section{Macular thickness in patients with choroidal neovascularization determined by RTA and OCT3: Comparative results}

${ }^{1}$ Instituto Oftalmológico de Alicante Vitreo-Retinal Unit Alicante, Spain

${ }^{2}$ Department of Ophthalmology Miguel Hernández University School of Medicine Alicante, Spain

Correspondence:

JA Montero Instituto Oftalmológico de Alicante Avenida de Denia 111. 03015 Alicante, Spain Tel: + 34902333344

Fax: + 34902333344

E-mail: msm02va@

wanadoo.es

Received: October 2003 Accepted: 20 October 2003 Published online: 23 April 2004

\begin{abstract}
Aims The authors conducted a study to compare retinal thickness in patients with choroidal neovascularization (CNV) using optical coherence tomography (OCT) and retinal thickness analyser (RTA).

Methods In all, 11 eyes from 11 patients with subfoveal choroidal neovascularization were examined with OCT and RTA.

Results Three patients could not be explored by RTA due to lack of fixation and high myopia. The mean foveal thickness was $289.9 \pm 92.1 \mu \mathrm{m}$ with OCT and 207.7 $\pm 60.8 \mu \mathrm{m}$ with RTA. The mean perifoveal thickness was 293.8 $\pm 46.3 \mu \mathrm{m}$ and $200.8 \pm 61.3 \mu \mathrm{m}$,

respectively. The maximal perifoveal thickness measured by OCT was $335 \pm 104.0 \mu \mathrm{m}$ and $316 \pm 86.8 \mu \mathrm{m}$ by RTA.

Conclusion OCT and RTA are able to detect increases in retinal thickness due to the presence of $\mathrm{CNV}$ and of fluid extravasation. They can be used in measuring retinal thickness in patients with $\mathrm{CNV}$, although measures are not comparable between both systems.

Eye (2005) 19, 72-76. doi:10.1038/sj.eye.6701400

Published online 23 April 2004
\end{abstract}

Keywords: retinal thickness analyser; optical coherence tomography; choroidal neovascularization

\section{Introduction}

Choroidal neovascularization $(\mathrm{CNV})$ is a leading cause of loss of central vision in developed countries and may appear associated with different ocular disorders, the most
JA Montero ${ }^{1}$ and JM Ruiz-Moreno ${ }^{1,2}$

common being age related macular degeneration (ARMD) and high myopia, and also after ocular inflammation and angioid streaks. ${ }^{1} \mathrm{New}$ advances in the treatment of $\mathrm{CNV}$, such as photodynamic therapy and transpupillary thermotherapy, have added new value to the determination of the activity of $\mathrm{CNV}$ through measurement of retinal thickness at the posterior pole. ${ }^{2,3}$

Retinal thickness analyser (RTA) is a new device for the study of the topography of the posterior pole of the eye. It projects $15-\mu \mathrm{m}$-wide slits of green light of $534 \mathrm{~nm}$, and records and digitizes light scattered back from the retina, and then localizing both interfaces and measuring the distance between them. These two peaks are later detected by a computer and the difference between them is measured, using this measure to map the posterior pole and calculate its thickness. ${ }^{4}$

Optical coherence tomography (OCT) provides high-resolution cross-sectional images of the retina permitting visualization of abnormalities in the retinal structure. It projects an infrared light of $820 \mathrm{~nm}$, which produces high-resolution (axial $10 \mu \mathrm{m}$, transversal $20 \mu \mathrm{m}$ ) interferometry cross-sectional images of the retina. ${ }^{5}$

In the present study, we have used the RTA and the new version of OCT (OCT3) to study changes in the vitreoretinal and chorioretinal surface in eyes with subfoveal CNV.

Patients, materials and methods

We have used the commercially available latest version of OCT (OCT3, Zeiss-Humphrey, San Leandro, CA, USA) and of RTA (Talia Technology Inc., Israel). 
In all, 11 patients with macular $\mathrm{CNV}$ associated to ARMD were studied using RTA and OCT3 by experienced operators. Measures were taken consecutively on the same day in order to make them comparable.

RTA was used to perform scans of a $6000 \times 6000 \mu \mathrm{m}^{2}$ area of the posterior pole centred in the fixation point (from $3000 \mu \mathrm{m}$ temporal to 3000 nasal to fovea, and from $3000 \mu \mathrm{m}$ superior to $3000 \mu \mathrm{m}$ inferior to fovea), using the posterior pole and peripapillary thickness function.

OCT3 performed $6 \mathrm{~mm}$ scans centred in the fovea (a circle $6000 \mu \mathrm{m}$ in diameter) using the fast macular scan function. Fixation was controlled by the video image of the central retina.

We have compared the OCT3 results of macular thickness in patients with CNV, with those of 40 healthy eyes (mean age 47.7 years range $20-78$ years; SD 18.2) from our database, which showed only mild refractive defects $(-4$ to $+2 \mathrm{D})$. For RTA we have used published data of healthy eyes. ${ }^{6}$ Linear correlation test was performed in order to compare the results by both procedures.

The results were compared in terms of absolute and relative thickness measured, and limitations and advantages of the procedures.

\section{Results}

Two patients could not be studied with RTA because of lack of central fixation. One patient could not have RTA performed because of high myopia (-12D). Five men and three women were fully studied, one eye in each patient, five right, and three left eyes. The mean age of patients was $73.5 \pm 9.6$ years (range $56-87$ years).

OCT provided good-quality images in all cases; meanwhile, RTA images showed partial lack of information in two cases $(25 \%)$.

The mean foveal thickness (MFT) as measured with OCT was $289.9 \pm 92.1 \mu \mathrm{m}$ and $207.7 \pm 60.8 \mu \mathrm{m}$ as measured with RTA $(r=0.954)$. The mean perifoveal thickness (MPT) was $293.8 \pm 46.3 \mu \mathrm{m}$ according to OCT and $200.8 \pm 61.3 \mu \mathrm{m}$ according to RTA $(r=0.920)$. The maximal perifoveal thickness (MT) measured by OCT was $335 \pm 104.0 \mu \mathrm{m}$ and $316 \pm 86.8 \mu \mathrm{m}$ by RTA $(r=0.707)$. Differences between both systems were more important in those patients with partial lack of information (Table 1, Figures 1-3).

Graphic topographic information was clear, intuitive, and self-explanatory in both instruments, although it was more marked with RTA (Figures $4 a$ and b).

OCT also provided cross-sectional images of the retina, which helped in determining whether the increase in retinal thickness was due to the presence of CNV itself or to the presence of accompanying fluid. Six patients showed active CNV expressed by the presence of fluid under the retina, and two showed lack of fluid by retinal thickening caused by the presence of CNV alone. Case 1 An 85-year-old lady with ARMD and good central fixation capability showed an MFT of $217.6 \mu \mathrm{m}$ with RTA and $302 \mu \mathrm{m}$ with OCT. Topographic images showed similar location of retinal thickening in both cases, mainly nasal and inferior to fixation. Crosssectional images by OCT, although, showed the presence of fluid under the retina proving the activity of CNV. Case 2 A 73-year-old man with ARMD showed an MFT of $226.3 \mu \mathrm{m}$ with RTA and $375 \mu \mathrm{m}$ with OCT. MPT was $224.2 \mu \mathrm{m}$ with RTA and $363.0 \mu \mathrm{m}$ with OCT. Central fixation was good. Topographic images showed in both cases an almost doughnut-shaped elevation of the posterior pole corresponding to $\mathrm{CNV}$. OCT images showed that retinal engorgement was caused by $\mathrm{CNV}$ with fluid accumulation under the retina (Figure 4c). Case 3 A 77-year-old man with ARMD and poor central fixation showed an MFT of $181.6 \mu \mathrm{m}$ with RTA and $202 \mu \mathrm{m}$ with OCT. MPT was $182.3 \mu \mathrm{m}$ with RTA and $241.5 \mu \mathrm{m}$ with OCT. Owing to poor central fixation RTA measures skipped over $75 \%$ of posterior pole giving way to misleading values. Topographic images showed retinal moderate thickening in the inferior nasal area with both techniques. OCT also indicated the presence of an atrophic CNV that produced no fluid around it,

Table 1 Macular thickness $(\mu \mathrm{m})$ measured by OCT and RTA

\begin{tabular}{|c|c|c|c|c|c|c|}
\hline & OCT MFT & RTA MFT & OCT MPT & $R T A M P T$ & OCT MT & RTA MT \\
\hline 1 & 358 & 275.4 & 328.5 & 224.2 & 431 & 334 \\
\hline 2 & 243 & 170.4 & 274 & 159.5 & 297 & 231 \\
\hline 3 & 202 & 185 & 241.5 & 182.3 & 252 & 355 \\
\hline 4 & 227 & 166.5 & 298.75 & 190.2 & 392 & 310 \\
\hline 5 & 236 & 178.3 & 282.2 & 181.5 & 289 & 220 \\
\hline 6 & 302 & 205.9 & 273.25 & 215.9 & 298 & 267 \\
\hline 7 & 268 & 154.2 & 227.75 & 154.5 & 244 & 266 \\
\hline 8 & 483 & 325.8 & 424.25 & 298.6 & 477 & 545 \\
\hline
\end{tabular}

OCT; Optical coherence tomography, MFT, mean foveal thickness, RTA, Retinal thickness analyzer, MPT, mean perifoveal thickness,m MT, maximal perifoveal thickness. 


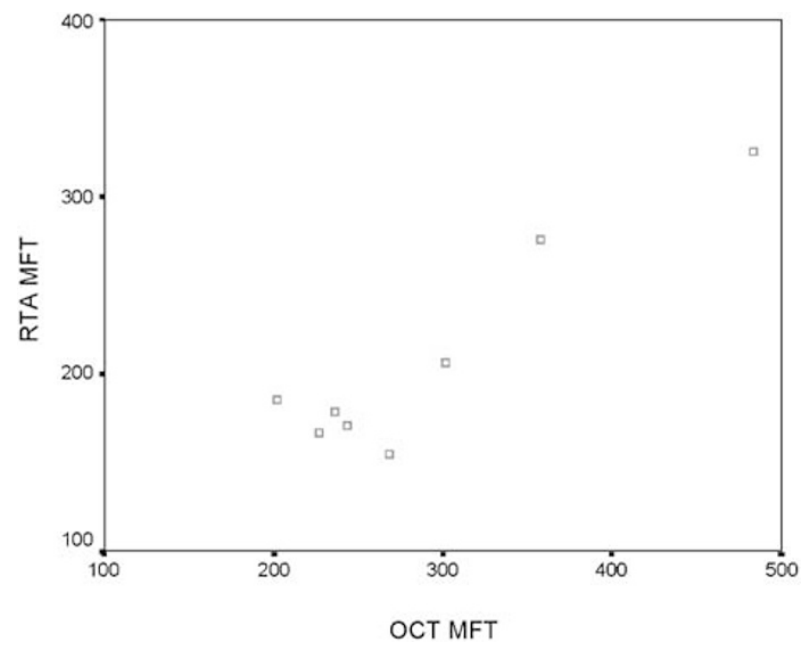

Figure 1 Scatter of MFT, $r=0.954$.

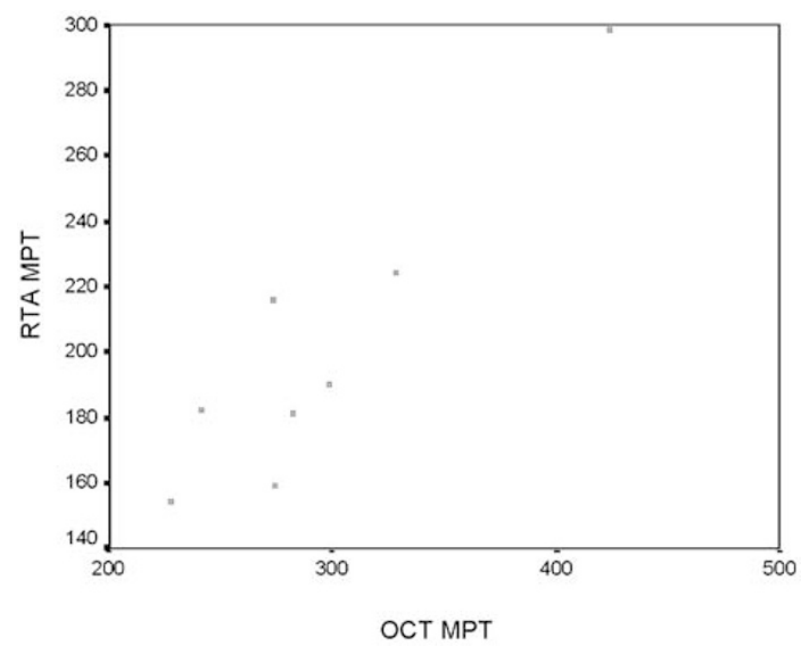

Figure 2 Scatter of MPT, $r=0.920$.

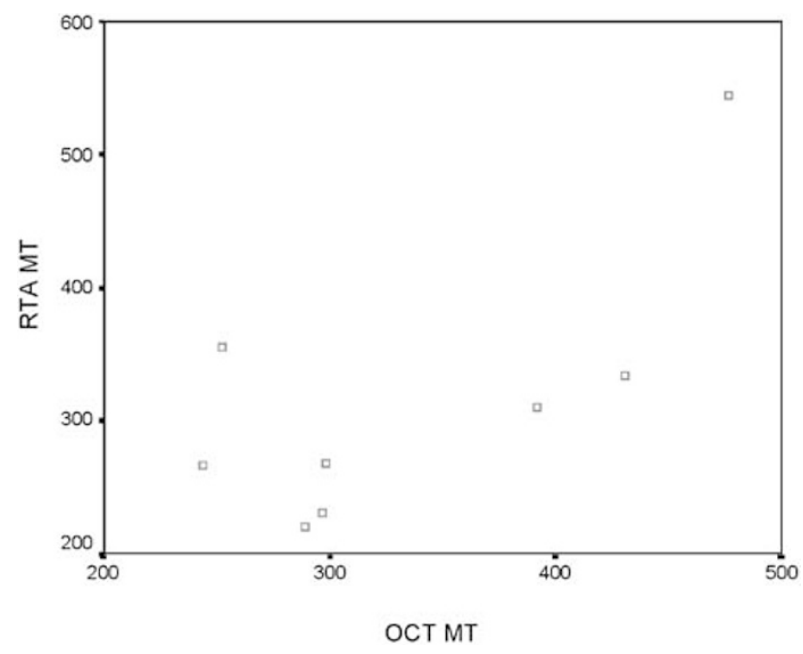

Figure 3 Scatter of MT, $r=0.707$. justifying the moderate thickening and poor fixation capability (Figure $4 \mathrm{~d}$ ).

\section{Discussion}

Retinal thickness in patients with CNV was increased in all measurements performed with both instruments. Thickness values were higher for OCT in absolute values but not as much when compared to values in healthy individuals. For RTA, MFT was $136.5 \%$ over healthy individuals $(152.1 \mu \mathrm{m})$ and MPT was $114.7 \%(175.0 \mu \mathrm{m})$. For OCT, they were $132.4 \%$ over healthy individuals $(219.8 \mu \mathrm{m})$ and $108 \%(272.5 \mu \mathrm{m})$, respectively. These results confirm those previously published on correlation between measures in both systems for healthy patients. ${ }^{7}$

Both instruments have previously proved to offer repetitive results concerning retinal pathology, especially for diabetic macular oedema. ${ }^{5,6,8}$

The differences found between both instruments may be related to several factors. First, the area considered by each device when measuring foveal or perifoveal thickness is not the same. RTA MFT takes into account the thickness of a circle of a $2000 \mu \mathrm{m}$ radius centred on the fixation point, whereas MPT is defined as the thickness in doughnut-shaped area around the fovea with an internal radius of $600 \mu \mathrm{m}$ and external radius of $2500 \mu \mathrm{m} .{ }^{6}$ OCT defines MFT as that of a circular area centred in the fixation point with a $1000 \mu \mathrm{m}$ radius, and for MPT it considers a doughnut-shaped area around the fovea with an internal radius of $500 \mu \mathrm{m}$ and external radius of $1500 \mu \mathrm{m}$.

Second, both systems take into account the distance between vitreoretinal and chorioretinal interfaces, by different procedures, using different wavelengths. Infrared light used by OCT allows imaging of structures placed deep in the retina in spite of the presence of vascular tissue such as $\mathrm{CNV}$ or haemorrhages.

Measurements of retinal thickness are obtained by using computer image processing techniques in both techniques, although OCT also allows manual measuring at selected points of the retina. RTA may show $\mathrm{CNV}$ by the elevation on the vitreoretinal surface. ${ }^{7}$

And third, lack of information related to certain areas of the posterior pole due to insufficient capability of fixation in RTA can also lead to errors in the evaluation of mean values.

The time required for image acquisition in RTA is variable and longer than required for OCT, since it requires several steps with changes in fixation. RTA changes the location of the fixation point in order to measure different parts of the posterior pole, forcing the follow movement of the eye. Thus, it demands a good fixation capability for an accurate measure, since data are discarded when fixation has not been good enough. On 
a

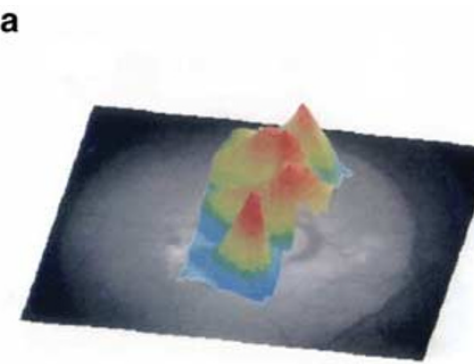

b

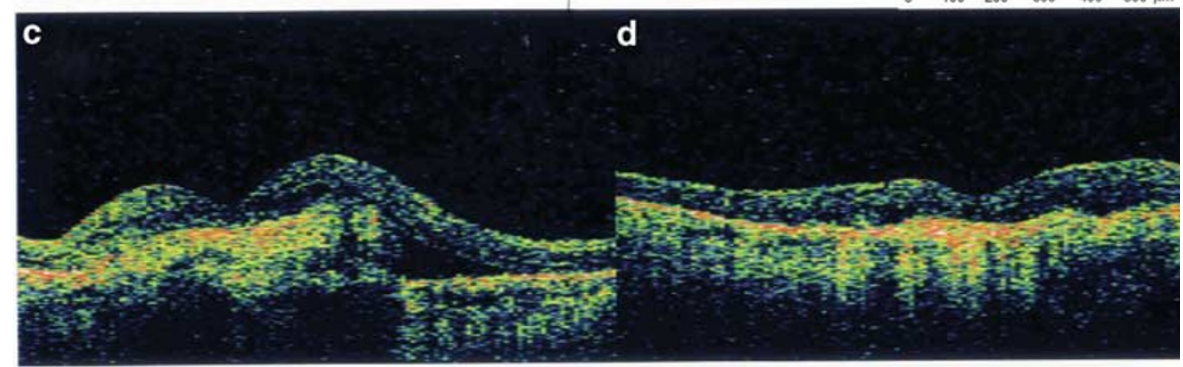

Figure 4 (a) RTA Topographic image shows highs and deeps of retinal surface in shapes and colours (case 2). (b) OCT topographic image in the same eye shows similar colour profile of the vitreoretinal interface. (c) CNV activity is proved by the presence of fluid deep in the retina (arrow). (d) This OCT image shows the presence of CNV with subretinal fibrosis, although no fluid accumulation can be seen.

the other hand, OCT makes a multiple scan in $1.92 \mathrm{~s}, 3.5$ or $6 \mathrm{~mm}$ in diameter, of six different meridians centred in a chosen point. Although both techniques require good fixation, the need for changing the fixation in order to change the scanned field with RTA makes it more difficult for the patient with poor fixation capability. OCT can use either internal or external fixation, and the operator can move the scan area and centre it in the fovea even if the patient is not able to follow the light.

One would expect that the fixation would be less of a problem with the RTA since the patient only needs to fix for about $0.3 \mathrm{~s}$ to acquire a scan. However, it is necessary for the patient to fix five times since five separate scans are taken that are then brought into registration by the software (or manually, if necessary). By comparison, the OCT requires $1.92 \mathrm{~s}$ of continuous fixation to acquire the six scan lines. As five scans are acquired, the time from start to finish is longer for RTA even though the total time the retina is being scanned by the instrument is less for RTA than for OCT. The quality of the data depends on the duration required for each scan, not the total time the patient is seated at the instrument for measurement.

The RTA has quality control procedures to reject data that are not satisfactory. This is not the case for OCT, which could produce unsatisfactory data without notifying the operator. Yet the appearance of discordant measures will make the operator aware of this. Both systems allow visualization of the fundus and manual centring of the data acquisition by the operator, if necessary.
RTA samples a large area of posterior pole by slits separated by less than $200 \mu \mathrm{m}$. This information is used to calculate values in at least 1280 locations from which the topographic map is generated. In contrast, only six 6$\mathrm{mm}$ lines of data are used to construct the topography with the OCT. However, no data are available for locations between the radial lines of data acquisition, and these are separated by as much as $1.5 \mathrm{~mm}$. The gaps in data are most prominent far from the fovea. Accordingly, while the unmeasured locations allegedly have values determined by interpolation, in reality most of the values are essentially fabrication. Furthermore, the centre of the map is constructed on the assumption that all the lines pass through the centre of the fovea. This assumption may not be met in some patients due to the difficulty in sustaining fixation for the entire data acquisition time.

Both systems are much limited by media opacities such as lens or posterior capsule opacities and by pupillary diameter, offering poor quality images that cannot be measured either manually or by the software. In terms of this aspect we have found no difference between them.

RTA has been used in the assessment of retinal thickness in diabetic patients with and without diabetic retinopathy, ${ }^{6-8}$ and proved to be reproducible. Although RTA may be useful for measuring diffuse retinal thickening, such as that of diabetic retinopathy, the more localized thickening and the poor fixation often found in patients with CNV may make it less sensitive in these patients. 
Both systems provide useful information in measuring retinal thickness in patients with posterior pole pathology. The limitations that RTA imposes due to fixation needs may work at the same time as a 'quality filter', improving the quality of the measures, and also reducing the number of patients who can benefit from this diagnostic procedure.

Cross-sectional images provided OCT, which gave important information to enable evaluation and to enable decision making in terms of patients with $\mathrm{CNV}$, since they inform about the indepth composition of the thickening, especially the presence of fluid and the shape, thickness, type, and size of $\mathrm{CNV} .^{9}$

In conclusion, OCT and RTA may provide important information in the study and follow-up of patients with $\mathrm{CNV}$, although thickness measures cannot be compared between both techniques or be considered in terms of absolute values since they are obtained by different means. The important relationship of RTA to fixation may make it less suitable for patients with foveal damage.

\section{References}

1 Sickenberg M, Schmidt-Erfurth U, Miller JW, Pournaras CJ, Zografos L, Piguet B et al. A preliminary study of photodynamic therapy using verteporfin for choroidal neovascularization in pathologic myopia, ocular histoplasmosis syndrome, angioid streaks and idiopathic causes. Arch Ophthalmol 2000; 117: 327-336.

2 Bressler NM. Treatment of age-related macular degeneration with photodynamic therapy (TAP) study group.

Photodynamic therapy of subfoveal choroidal neovascularization in age-related macular degeneration with verteporfin Two-year results of 2 randomized clinical trials TAP report 2. Arch Ophthalmol 2001; 119: 198-207.

3 Newsom RSB, McAllister JC, McHugh JDA. Transpupillary thermotherapy (TTT) for the treatment of choroidal neovascularisation. Br J Ophthalmol 2001; 85: 173-178.

4 Zeimer R, Shahidi M, Mori M, Zou S, Asrani S. A new method for rapid mapping of the retinal thickness at the posterior pole. Invest Ophthalmol Vis Sci 1996; 37: 1994-2001.

5 Hee MR, Puliafito CA, Duker JS, Reichel E, Coker JG, Wilkins JR et al. Topography of diabetic macular edema with optical coherence tomography. Ophthalmology 1998; 105: 360-370.

6 Konno S, Akiba J, Yoshida A. Retinal thickness measurements with optical coherence tomography and the scanning retinal thickness analyzer. Retina 2001; 21: 57-61.

7 Fritsche P, van der Heijde R, Suttorp-Schulten MS, Polak BC. Retinal thickness analysis (RTA). An objective method to assess and quantify the retinal thickness in healthy controls and in diabetics without diabetic retinopathy. Retina 2002; 22: 768-771.

8 Goebel W, Kretzchmar-Gross T. Retinal thickness in diabetic retinopathy. A study using optical coherence tomography (OCT). Retina 2002; 22: 759-767.

9 Rogers AH, Martidis A, Greenberg PB, Puliafito CA. Optical coherence tomography findings following photodynamic therapy of choroidal neovascularization. Am J Ophthalmol 2002; 134: 566-576. 